\title{
Ayahuasca: el encuentro de dos paradigmas.
}

\author{
Ayahuasca: the meeting of two paradigms. \\ Daniel E. Rojas-Bolívar ${ }^{1}$ \\ RESUMEN
}

La ayahuasca es una liana que crece en la Amazonía y con la cual se prepara una sustancia con potentes propiedades psicoactivas, también denominada ayahuasca. Esta sustancia está ligada con la cosmovisión de los pueblos amazónicos que la consideran una planta espiritual y medicinal por las visiones y percepción de los efectos que produce. Las investigaciones en laboratorio muestran que la ayahuasca contiene derivados betacarbolínicos como la harmina y derivados triptamínicos como la N,N-dimetiltriptamina (DMT), moléculas que intervienen en determinados fenómenos psicodislépticos de tipo alucinatorio -visiones de seres sobrenaturales- junto a alteraciones de la conciencia del yo descritas como una sensación de muerte y renacimiento que pueden llevan al usuario de la ayahuasca a un estado de éxtasis espiritual y bienestar. Estos efectos han sido descritos en la literatura y se ha considerado el potencial terapéutico de la ayahuasca frente a trastornos del estado de ánimo y problemas adictivos en los cuales se han reportado algunos resultados favorables. Sin embargo, los efectos psicoactivos que genera la ayahuasca son complejos debido a sus aspectos subjetivos, sociales y culturales y por consiguiente son difíciles de abordar objetivamente en el marco del paradigma científico actual. Por tales motivos resulta necesario el replanteamiento de formas alternativas de investigación que puedan interpretar la subjetividad presente en la experiencia psicoactiva por uso de ayahuasca.

PALABRAS CLAVE: Banisteriopsis, antropología médica, psiquiatría.

\section{SUMMARY}

Ayahuasca is a vine that grows in the Amazon, used to prepare a substance with high psychoactive properties also called ayahuasca. This substance is linked with the worldview of the Amazonian people who consider it a spiritual and medicinal plant due to the views and perceptions of the effects it produces. Laboratory research shows that ayahuasca contains $\beta$-carbolinicderivates, such as harmineand tryptaminederivatives such as N,N-dimethyltryptamine (DMT), which are molecules involved in psychoactive processes related to visions of supernatural beings and the sense of death and rebirth that leads to a state of higher spirituality and wellness. These effects were studied to evaluate the therapeutic potential of ayahuasca in mood disorders and addictions, and favorable results were found. However, the psychoactive effects of ayahuasca are accompanied by subjective, social and cultural features which generate difficulties for an objective approach to the experience under the positivist paradigm. For these reasons it is necessary to rethink alternative forms of research that can interpret the subjectivity present in the psychoactive experience and, with it, a change in training and in the practice of medicine.

KEYWORDS: Banisteriopsis, medical anthropology, psychiatry.

Al Dr. Carlos Alberto Seguín, in memoriam.

1 Estudiante. Facultad de Medicina de San Fernando. Universidad Nacional Mayor de San Marcos. Lima, Perú. 


\section{INTRODUCCIÓN}

La medicina contemporánea busca soluciones alternativas frente a los problemas de salud y para ello adopta experiencias de diferentes culturas con el propósito de conocer sus recursos terapéuticos y aplicarlos a nuevos contextos. Es así como se ha tomado interés en una liana de la Amazonía que, al ser preparada con otras plantas, produce una bebida de importantes propiedades psicoactivas utilizada con fines medicinales y espirituales en las comunidades amazónicas. Esta sustancia es llamada también ayahuasca (1). Su uso está extendido en las diferentes comunidades nativas de la selva amazónica y tiene un importante significado cultural y espiritual que no se puede desligar de la imagen del curandero, el intermediario entre el mundo de lo sobrenatural y el mundo de los humanos (2).

A nivel bioquímico, se ha encontrado en el preparado de la ayahuasca moléculas tales como la harmina y la N,N-dimetiltriptamina (DMT), las cuales tienen un potente efecto psicoactivo y por ende cumplen un rol clave en los efectos deseables del brebaje (3), y que podrían tener además implicaciones en los mecanismos bioquímicos de ciertas patologías psiquiátricas. La ayahuasca está siendo considerada, a nivel experimental, como una opción terapéutica frente a ciertos trastornos psiquiátricos de tipo ansioso y depresivo, además de problemas de adicción a sustancias (4-6). Sin embargo, la importante carga subjetiva, así como el contexto cultural en el que se consume la ayahuasca, dificulta un abordaje estrictamente objetivo con la consiguiente posibilidad de producir resultados más o menos sesgados.

Esta realidad cuestiona los alcances del paradigma epistemológico que rige muchas de las investigaciones y sus normas protocolarias en las ciencias y el sistema de salud. En este contexto se observa entonces una contradicción entre dos formas de medicina: la medicina académica y la medicina folklórica o tradicional.

Frente a los problemas planteados resulta necesario el desarrollo de un nuevo paradigma que incluya una visión transdisciplinaria que abarque no solo los aspectos objetivos cuantificables sino también la subjetividad y los factores culturales presentes en este fenómeno. Y quizá también el desarrollo de una nueva forma de medicina que articule la medicina académica y medicina tradicional en un marco de respeto e igualdad cultural donde el objetivo final sea el bienestar y plenitud del ser humano.

\section{La Ayahuasca}

La ayahuasca es una liana leñosa del género Banisteriopsis que pertenece a la familia Malpighiaceae. Es una planta de tipo arbustiva, trepadora, de ramas grises o parduzcas que pueden llegar a tener un diámetro de diez centímetros. Se envuelve en los árboles y se enrolla sobre sí misma formando una especie de cuerda. Se encuentra distribuida como planta silvestre en la cuenca amazónica, en el oeste de Brasil, el nordeste del Perú y en zonas de Colombia y Ecuador $(1,7)$. Este género cuenta con más de cien especies de los cuales una de las más representativas es la Banisteriopsis caapi (7). Cuando cocida por tiempo prolongado junto a las hojas de la "chacruna" (Psychotria viridis), se obtiene la bebida denominada también ayahuasca, que tiene potentes efectos psicoactivos y cuyo uso es ritual y ceremonial en diversos pueblos amazónicos (2). El término ayahuasca deriva de dos palabras del idioma quechua: "aya" que significa muerto y "huasca" que significa soga. Por eso se le conoce como "la soga de los muertos", "la enredadera de las almas" o "el ombligo de la tierra". Tiene también otros nombres según las diversas regiones: yagé, caapi, shillinto, natéma, pinde, purga-huasca, entre otros $(1,7,8)$.

La ayahuasca está estrechamente ligada con la cosmovisión de las comunidades indígenas amazónicas $(7,9)$. Por ejemplo, en el Perú, es considerada una "planta maestra" ya que al ser "morada de espíritus", permite entrar en contacto con el mundo de lo sobrenatural. En este contexto, el brebaje de ayahuasca facilita al curandero ejercer su poder sanador, es decir, diagnosticar, encontrar las causas de la enfermedad y además establecer la cura respectiva. La ayahuasca es el agente que "dicta" al curandero los cantos ceremoniales ("ícaros") para proteger a él y al paciente (1). En el caso de la tribu de los Tukano en Colombia, el uso del yagé está enmarcado en un contexto ceremonial en el que, por medio de cantos y danzas, el curandero y las personas participantes llegan a un estado modificado de conciencia caracterizado por la presencia de visiones de un estado de muerte y renacimiento y donde aparecen visiones de seres míticos, ancestrales y sobrenaturales. Esta experiencia es esencial en la vida de las personas que han tomado el yagé porque implica el renacimiento en forma de una nueva persona (2). Entre los Runas de Ecuador, el curandero es un intermediario entre el mundo espiritual, el mundo de la naturaleza y el mundo de los humanos. A través de la ingestión de un "agente transformativo" como la ayahuasca, el curandero puede tener contacto con lo sobrenatural y relacionarse con las almas e 
inclusive tomar formas de animales, en particular de felinos (10). En las comunidades de los Ese'ejja en Bolivia se prepara el xono, combinación de ayahuasca con otras dos plantas, el ziño y la zamarquita. Su uso está enmarcado en un contexto sacralizante en el que la comunidad participa (11). En Brasil, además del uso ceremonial de la ayahuasca en las comunidades indígenas, se utiliza también en comunidades mestizas. Este uso llegó a institucionalizarse en forma de religiones como la del Santo Daime y la União do Vegetal, que integra creencias católicas y de otras religiones o cultos espirituales $(6,12)$. En Brasil, cabe señalar que las religiones mencionadas están muy difundidas $\mathrm{y}$, de hecho, parecen estarse expandiendo por varias regiones del mundo, tal como lo documentan Labate y cols. (12) en una revisión exhaustiva del tema; Es importante señalar que en todos los casos mencionados, el uso de la ayahuasca otorga al curandero facultades para desarrollar un poder de curación. De hecho, la ayahuasca es ya considerada en estos ámbitos como una planta medicinal $(1,13)$.

\section{Farmacología de los componentes de la ayahuasca.}

Los principales componentes químicos que contiene la Banisteriopsis caapi son alcaloides del grupo betacarbolínico tales como harmina, harmalina, tetrahidroharmina y harmalol, que tienen una función de inhibición de la mono-amino oxidasa (IMAO). Por su parte, la Psychotria viridis contiene principalmente un derivado triptamínico con propiedades psicoactivas: la N,N-dimetiltriptamina (DMT) (5).

Los alcaloides betacarbolínicos como la harmina se caracterizan por ser potentes inhibidores de la enzima mono-amino oxidasa (IMAO). Esta enzima actúa en el metabolismo de las catecolaminas, la dopamina y la triptamina, de tal manera que permite que las concentraciones de estos neurotransmisores se incrementen; además, estos alcaloides betacarbolínicos evitan que la mono-amino oxidasa (MAO) hepática degrade la DMT (14). Dicho de otro modo, la harmina y sus derivados favorecen una mayor concentración de los neurotransmisores mencionados arriba y evitan que la DMT sea degradada en el hígado. Los estudios han mostrado que la harmalina tiene una gran afinidad por la enzima DYRK1A, una moderada afinidad por la 5-hidroxitriptamina 1A (5-HT1A), 5-HT2A, los receptores de imidazolina $\mathrm{I} 2$ y poca afinidad por los receptores 5-HT2C y los receptores de transportadores de dopamina $(15,16)$. Diversas investigaciones muestran que los derivados betacarbolínicos tienen también propiedades psicoactivas (3). Así, Anderson (5) recoge las experiencias de autores que han trabajado con la harmina y que sostienen que tiene efectos psicodélicos (5).

Otro componente químico importante, la $\mathrm{N}, \mathrm{N}$ dimetiltriptamina (DMT), es un metabolito derivado de la triptamina de potente efecto psicoactivo. Esta propiedad se debe principalmente a la afinidad que tiene por los receptores 5-HT2A al ser un agonista serotoninérgico (3). Sin embargo, la vía serotoninérgica no es la única vía de acción de la DMT. Estudios experimentales en ratones evidencian que tiene acción sobre otros receptores tales como el TAAR1 y el receptor sigma-1, lo cual significa que, además de una actividad serotoninérgica, la DMT tiene también una acción dopaminérgica (15).

\section{Efectos de la ingestión de ayahuasca.}

Los efectos que causa el consumo de ayahuasca pueden ser descritos en tres fases (17). En primer lugar se evidencian síntomas de tipo neurovegetativo tales como mareos, salivación, temblor fino generalizado, náuseas, así como un peristaltismo intensificado acompañado de dolores tipo cólico y vómitos, además de un incremento moderado de la frecuencia cardiaca y de la presión arterial $(18,19)$. En este periodo también comienzan los efectos psicodélicos y la persona entra a un estado alterado de consciencia. En la segunda fase, estos efectos se intensifican con visiones sobre diferentes temas de índole cósmica y mítica. Finalmente, en la tercera fase, la persona entra en un profundo estado de introspección con libre asociación de ideas de intenso tono emocional que se conservan intensamente en la memoria (20).

Los efectos psíquicos del consumo de la ayahuasca son diversos. Los primeros estudios realizados consistieron en los reportes de las experiencias percibidas por la autoadministración de esta sustancia. Villavicencio refirió haberla ingerido en Ecuador y haber visualizado "alucinaciones maravillosas". Reimburg describe efectos neurovegetativos en la primera ingesta y sobreexcitación con visiones luminosas, a dosis mayores (21), mientras Halpern mencionó una sensación semejante a la levitación (22). En ensayos con humanos realizados en el Perú por Ríos y cols.(18), se describe que con la ingestión de ayahuasca las personas mostraron alteraciones en la percepción tales como sensación de movimiento aun estando en reposo, y disminución del umbral auditivo por lo que los ruidos resultaban muy molestos.

Además presentaban ilusiones, alucinaciones y sinestesias. Respecto a éste punto cabe señalar que los 
participantes refieren que pueden "escuchar colores" (sinestesia auditivo-óptica) o que sienten la música como "formas que ondulan rítmicamente, que tienen aspecto de extrañas nubes y en las que está flotando el propio cuerpo" (sinestesia auditiva-óptica-cenestésica) (18). Asimismo, se aprecian fenómenos visuales alucinatorios de tipo geométrico y caleidoscópico, así como la aparición de animales y seres sobrenaturales.

La persona entra en un estado de tranquilidad y experimenta la sensación de la "manifestación de lo divino" (23). Por su parte, Strassman y cols. (24) hallaron que la ayahuasca produce efectos tales como excitación y euforia, así como un estado de contemplación precedido de momentos de confusión.

Los participantes refieren que surgen en ellos nuevas perspectivas acerca de sí mismos y su entorno, lo que origina una conmoción en sus bases intelectuales y emocionales cotidianas. Una investigación similar se realizó en Brasil, evaluándose los efectos del consumo de ayahuasca en un contexto ceremonial. En este estudio se distinguen principalmente las sensaciones de serenidad y resolución frente a los factores que previamente causaban estrés, una mayor vivacidad, así como regocijo y satisfacción (23). Sin embargo, se reporta también preocupaciones o angustia ligadas a las vivencias percibidas. Por otro lado, Riba y cols. (14) reportaron en un ensayo clínico efectos subjetivos entre los que resaltan las sensaciones de euforia y bienestar, así como alteraciones en las percepciones visuales, contenido cognitivo y labilidad emocional (14). Debe señalarse que estos hallazgos fueron resultados de la aplicación de instrumentos psicométricos y por ende ceñidos a un marco cuantitativo, situación que no sucede en el estudio de Kjellgren y cols., (25) quienes a través de un estudio cualitativo con análisis fenomenológico hallaron importantes categorías en el análisis de la experiencia psicoactiva; destacan entre ellas un cambio en la concepción del mundo y una reorientación en el curso de vida expresada como variaciones en los intereses de los participantes y en una búsqueda de desarrollo personal. Los participantes manifestaron que patrones psicológicos tales como los temores disminuyeron, en tanto que su afectividad positiva se incrementó.

Reportaron además otros efectos como el aumento en la creatividad, mejora de habilidades cognitivas y desarrollo de un benéfico "estado espiritual". Estos resultados se mantienen en la investigación de Santos y cols. (26) que reporta disminución del estado de ansiedad en las personas que consumen ayahuasca, mientras que Frecska y cols., mencionan un aumento de respuestas originales en una prueba psicométrica que evalúa la creatividad (27). Por otra parte, en un estudio longitudinal, Bouso y cols. no encontraron evidencia de psicopatología a largo plazo en personas que consumen ayahuasca (28).

Los estudios citados demuestran que la ayahuasca genera un cambio importante en las personas en relación a su concepción de la vida, a su existencia y el entorno. En otras palabras, se generan cambios a nivel perceptivo, afectivo, cognitivo, volitivo, conductual y existencial.

Considerando lo mencionado y teniendo en cuenta que no se ha reportado evidencias científicamente comprobadas de que el consumo de la ayahuasca genere efectos adversos perjudiciales $(29,30) \mathrm{ni}$ que sea una sustancia potencialmente adictiva (5), numerosos científicos proponen a la ayahuasca como una opción terapéutica frente a desórdenes psiquiátricos tales como la ansiedad, la depresión y la adicción a sustancias $(4,6,23,26)$.

Sin embargo, existe una resistencia a la instauración de este tipo de terapias, esencialmente porque resulta necesaria una mayor investigación sobre los efectos terapéuticos así como sus efectos nocivos. No obstante, nace la interrogante de si existe un rechazo subyacente del modelo médico vigente hacia la medicina folklórica.

\section{La Ayahuasca: El encuentro de dos paradigmas.}

El análisis de los efectos terapéuticos de la ayahuasca resulta tan complejo como las visiones que produce, ya que especialidades como la psiquiatría, las neurociencias y la antropología médica se entrelazan e interconectan para tratar de explicar porqué la ayahuasca podría ser una forma de medicina y cuál sería su mecanismo de acción. Las investigaciones farmacológicas brindan bastante información sobre los efectos biológicos de esta sustancia, pero permanece la interrogante de si todos los efectos se pueden explicar por la acción de la DMT sobre los receptores serotoninérgicos. La toma de ayahuasca está enmarcada en un contexto cultural cuyas concepciones y tradiciones pueden aportar como adyuvantes terapéuticos a esta sustancia. Seguín describe los procedimientos usados por los curanderos en la medicina folklórica (31). En su descripción menciona el uso de las maniobras mágicas, las maniobras psicológicas y la ingestión de sustancias psicoactivas 
como la ayahuasca. Las maniobras mágicas consisten en las invocaciones a los espíritus favorables así como rechazo a los que resultan dañinos. Las psicológicas incluyen la expectativa positiva que presenta la persona que busca al curandero (31). Es aquí donde resulta importante analizar el papel de uno de los elementos más importantes en el estudio de los efectos causados por la ayahuasca en su contexto cultural: el curandero o chamán, un intermediario entre el mundo de lo sobrenatural y el mundo de los humanos (2). Este personaje acostumbra tener un yo fuerte, con una importante capacidad de sugestión. Para ello requiere una preparación rigurosa que es lograda a través de privaciones, ayunos y abstinencia sexual por tiempos prolongados (32). Con tal preparación, el curandero se desarrolla y se convierte en una persona madura, con actitudes paternales, seguras y bondadosas. Así, ejerce una fuerte interacción con las personas que lo buscan y quienes incluso le guardan más confianza que al médico. Esto se debe a que los curanderos se encuentran social, cultural y, sobre todo, afectivamente vinculados a los pacientes, lo cual favorece su acción terapéutica. Seguín denomina a este tipo de vínculo "eros terapéutico", y lo define como "el amor por el paciente $\mathrm{o}$, mejor dicho por la persona del paciente, con lo que se quiere expresar que no se trata de una forma de amor "humanitario" que el médico debe sentir por el enfermo como enfermo, sino de un movimiento auténtico hacia el individuo único que se halla ante él -que es éste y no otro- y que no es "enfermo" sino un hombre." (33).

Este tipo de vínculo es muy notorio en los curanderos, más aún, como decíamos, que con los médicos. Debe considerarse la comunicación horizontal que existe entre el curandero y el paciente, que comparten un contexto socio-cultural y "hablan el mismo idioma". Además, el curandero muestra capacidades de atención y escucha que el paciente requiere, hecho que sucede con bastante menos frecuencia en el medio hospitalario. Es importante señalar también cómo los curanderos han desarrollado empíricamente formas de terapia de grupo, terapias familiares y, sobre todo, una forma de cambiar las relaciones interpersonales del paciente y con ello romper círculos viciosos que prolongaban las enfermedades $(34,35)$.

Las descripciones de las maniobras mágicas, las maniobras psicológicas y la ingestión de sustancias psicoactivas fueron detalladas por Lemlij y Hernández (21) quienes mostraron cómo el curandero a través de cantos y silbidos invoca a los espíritus de las "plantas maestras". Estas canciones, que se caracterizan por su carácter regresivo, se asemejan a canciones de cuna y vienen con exhortaciones enérgicas o tiernas, repetitivas, con una inseguridad tonal que se hace ascendente hasta llegar a tonalidades más elevadas (melodías escalonadas), de ritmo acelerado, velocidad e intensidad cambiante. Con estas canciones el curandero resuelve la angustia y ansiedad de los pacientes que acuden a su ceremonia (21). Resulta importante tener en cuenta la posible influencia terapéutica de los cantos ceremoniales. Algunas investigaciones muestran los efectos beneficiosos de la musicoterapia sobre el estado de ánimo (36), aún cuando todavía muestran vacíos metodológicos. Además, existen pocos estudios experimentales que abordan este tema (37).

Por otra parte, como se mencionó anteriormente, los participantes en las ceremonias de toma de ayahuasca experimentan una manifestación de lo divino y cambian sus percepciones sobre la espiritualidad, lo cual puede tener una relación con el bienestar la de las personas (38). En este sentido se realizaron investigaciones que, precisamente, sugieren una relación entre espiritualidad y bienestar pero que, al igual que en el caso de la musicoterapia, encuentran dificultades en el desarrollo de un método más objetivo y libre de sesgos (39). Podemos afirmar que existe evidencia que demuestra los efectos terapéuticos de las prácticas mencionadas de la medicina tradicional. Sin embargo, a pesar de las evidencias mostradas, el marco epistemológico de la medicina occidental discute los resultados por la subjetividad presente en éstas y los considera, por lo tanto, controversiales.

Estas observaciones nos muestran la contradicción que existe entre el modelo de la medicina tradicional, que incluye elementos como música, danzas, psicodelia y espiritualidad, es decir, un modelo en el que predomina la subjetividad (1). Mientras tanto, el modelo de medicina occidental se caracteriza por ser analítico, reduccionista, positivista y universalista y por tender a aislar el cuerpo humano de dimensiones como lo social, lo afectivo y espiritual (40).

La aparición de la ayahuasca en el ámbito de la medicina impacta sobre el modelo establecido de saludenfermedad-atención y genera una contradicción con el paradigma médico occidental; a punto de partida de esta contradicción se genera un tabú científico que viene a ser la prohibición de tomar en serio algunos conocimientos que están fuera de sus límites (41).

Esta contradicción se manifiesta en la conceptualización más bien negativa que la medicina 
occidental tiene de las sustancias con efectos psicoactivos del tipo aquí descrito. Además, deja de lado la importancia cultural y simbólica que tienen estas sustancias en la cosmovisión de las comunidades donde se usan de manera ceremonial. Desde la perspectiva del otro paradigma, a estas sustancias se les denomina "enteógenos" ("lo que genera a Dios en nuestro interior") (7). Es evidente la contradicción entre los dos paradigmas. Se deja de lado el término "alucinógeno", con fuerte connotación negativa. Algunos autores proponen el término "lucidógeno" (42), que considera a los efectos psicoactivos como un estado modificado de conciencia en el cual hay una percepción aumentada de la realidad.

Es en esta contradicción donde se observa que la ayahuasca desafía el paradigma médico occidental ya que siendo una sustancia "alucinógena" tiene efectos terapéuticos que son demostrados en las rigurosas condiciones del laboratorio experimental, pero que están insuficientemente comprendidos al ser desligada de su contexto cultural y ceremonial. No es suficiente estudiar las plantas medicinales en los laboratorios de bioquímica o farmacología. Es necesario comprender y reconocer la naturaleza de la medicina tradicional que, apoyada en los entéogenos, busca un tratamiento integral de la persona. Asimismo, es necesario que los profesionales de la salud tengan una actitud abierta y eviten considerar la experiencia psicoactiva como una actitud equivocada o una actitud de riesgo (43). Esta forma de terapia se diferencia de la visión académica por un factor esencial: el lugar de la subjetividad. Por este motivo surge la necesidad de un replanteamiento de los paradigmas epistemológicos hacia un nuevo paradigma donde se reconozca la importancia de la subjetividad en los procesos de salud-enfermedadatención y en el cual la investigación analice no solo los aspectos positivos y cuantificables de la realidad sino que también interprete los aspectos subjetivos y culturales del ser humano.

La consideración de la subjetividad en las ciencias de la salud trae como ventaja una amplitud de perspectiva respecto a los procesos de saludenfermedad-atención que se traduce en una visión concreta, dinámica y comprensiva del problema (44). Ya no sería el abordaje de la enfermedad, sino el conocimiento del ser humano y la enfermedad.

Entonces, así como se manifiesta un renacimiento y una reorientación en el curso de vida luego de la experiencia con la ayahuasca, es necesaria una reorientación en la metodología de la investigación donde se desarrollen más estudios interdisciplinarios y transdisciplinarios para abordar la compleja realidad que se observa en los procesos de salud-enfermedadatención con el uso de plantas psicoactivas.

Mas, no es suficiente una reorientación metodológica de la investigación. Es necesaria una real consideración de los aportes de la medicina tradicional en un marco de igualdad y respeto a las diferentes culturas y sus métodos terapéuticos. Para ello se necesita tanto de la enseñanza de la medicina tradicional en las universidades como, sobre todo, la articulación de la medicina académica con la medicina tradicional en los sistemas de salud, con el fin de asegurar el bienestar del ser humano cmo totalidad, no solamente en un plano biológico, psicológico y social sino también en un plano cultural, formativo y espiritual.

\section{CONCLUSIONES}

Los importantes componentes subjetivos y culturales, estrechamente enlazados con el consumo de ayahuasca, reciben un abordaje incompleto por parte de la medicina occidental cuyo enfoque es notoriamente positivista. Este proceso evidencia un encuentro de dos paradigmas: por una parte, la medicina tradicional con sus componentes subjetivos y la medicina occidental con su abordaje exento de subjetividad. En este contexto, es necesario el desarrollo de un nuevo paradigma que contemple tanto los aspectos objetivos y técnicos propios de la medicina occidental como los aspectos subjetivos, culturales y espirituales de la medicina tradicional con el fin de promover la salud y bienestar integrales del ser humano.

\section{Correspondencia: Daniel E. Rojas-Bolívar}

Dirección: Sector 3, Grupo 27, Manzana H, Lote 23.

Villa El Salvador

Código postal: Lima 42

Correo electrónico: danielrobo871@gmail.com

\section{REFERENCIAS BIBLIOGRÁFICAS}

1. Hurtado L. Perfil cultural del shamanismo de la Amazonía peruana. En: Alva W, Polía M, Chávez F, (editores). Shamán: la Búsqueda. Córdoba: Imprenta San Pablo; 2000. p. 225-69.

2. Furst P. La Ibogaína y la enredadera de las almas: Del ritual de los bosques tropicales a la psicoterapia. Alucinógenos y Cultura. 1 ed. México DF: Fondo de Cultura Económica; 1980. p. 83-100.

3. Picabea L. Aspectos químico-farmacológicos de los 
alucinógenos utilizados en los ritos por los chamanes de la Amazonía peruana. En: Alva W, Polía M, Chávez F, editors. Shamán: la Búsqueda. Córdoba: Imprenta San Pablo; 2000. p. 272-84.

4. Anderson BT. Ayahuasca as Antidepressant? Psychedelics and Styles of Reasoning in Psychiatry. Anthropology of Consciousness. 2012; 23(1): 44-59.

5. Fábregas JM, González D, Fondevila S, Cutchet M, Fernández X, Barbosa PCR, et al. Assessment of addiction severity among ritual users of ayahuasca. Drug Alcohol Depend. 2010; 111(3): 257-61.

6. Guimarães R, Carvalho C, Holanda A. Ayahuasca e redução do uso abusivo de psicoativos: eficácia terapêutica? Psicologia: Teoria e Pesquisa. 2006; 22 (3): 363-70.

7. Llamazares AM, Martínez C. Principales plantas sagradas de Sudamérica. En: Llamazares AM, Martínez C, (editores). El Lenguaje de los Dioses: Arte, Chamanismo y Cosmovisión Indígena en Sudamérica. 1 ed. Buenos Aires: Editorial Biblos; 2004. p. 259-66.

8. Palacios J. Plantas Medicinales nativas del Perú-I. 1 ed. Lima: CONCYTEC; 1993.

9. Eddowes-Villarán J. Las plantas maestras en el desarrollo de la medicina intercultural. In: SolanoMendoza L, editor. VIII Congreso Mundial de Medicina Tradicional, Alternativa, Complementaria y Cuántica: Libro de Resúmenes. Lima: CIMT; 2012. p. 57-61.

10. Kohn E. El mal runa en perspectiva social y simbólica. La Cultura Médica de los Runas de la Región Amazónica Ecuatoriana: Ediciones Abya-Yala; 1992. p. 11-34.

11. Pages Larraya F. [The meaning of the use of ayahuasca among the Chama (Ese'ejja) natives of Eastern Bolivia: a transcultural study]. Acta Psiquiatr Psicol Am Lat. 1979; 25(4): 253-67.

12. Labate BC, Rose IS, Santos RG. Ayahuasca Religions: A Comprehensive Bibliography \& Critical Essays. Santa Cruz CA: MAPS; 2008.

13. Doyle R. Healing with Plant Intelligence: A Report from Ayahuasca. Anthropology of Consciousness. 2012; 23 (1): 28-43.

14. Riba J, Barbanoj MJ. Bringing ayahuasca to the clinical research laboratory. J Psychoactive Drugs. 2005; 37: 219-30.

15. Brierley DI, Davidson C. Developments in harmine pharmacology - implications for ayahuasca use and drug-dependence treatment. Prog Neuropsychopharmacol Biol Psychiatry. 2012; 39 (2): 263-72.

16. Dos Santos RG, Valle M, Bouso JC, Nomdedéu JF, Rodríguez-Espinosa J, Mcllhenny $\mathrm{EH}$, et al. Autonomic, neuroendocrine, and immunological effects of ayahuasca: a comparative study with d-amphetamine. J Clin Psychopharmacol. 2011; 31
(6): 717-26.

17. Naranjo P. Hallucinogenic plant use and related indigenous belief systems in the Ecuadorian Amazon. J Ethnopharmacol. 1979; 1(2): 121-45.

18. Ríos Ó, Hernández M, Lemlij M, León F. Estudios sobre la harmina y el ayahuasca I. Descripción fenomenológica y evaluación clínica del psicosíndrome. Revista de Ciencias Psicológicas y Neurológicas. 1964;1(2):211-33.

19. Strassman R, Qualls C. Dose-response study of $\mathrm{N}, \mathrm{N}$-dimethyltryptamine in humans. I. Neuroendocrine, autonomic, and cardiovascular effects. Arch Gen Psychiatry. 1994; 51 (2): 85-97.

20. Laqueille X, Martins S. L'Ayahuasca: clinique, neurobiologie et ambiguïté thérapeutique. Annales Médico-psychologiques, revue psychiatrique. 2008; 166(1): 23-7.

21. Lemlij M, Hernández M. Los psicodislépticos en la selva peruana. Ayahuasca y toé en psicoterapia curanderil. Anales del Servicio de Psiquiatría. 1967; 9 (4):114-27.

22. Sanchez-Ramos JR. Banisterine and Parkinson's disease. Clin Neuropharmacol. 1991; 14 (5): 391402.

23. Barbosa PCR, Giglio JS, Dalgalarrondo P. Altered states of consciousness and short-term psychological after-effects induced by the first time ritual use of ayahuasca in an urban context in Brazil. J Psychoactive Drugs. 2005; 37 (2): 193-201.

24. Strassman RJ, Qualls CR, Uhlenhuth EH, Kellner R. Dose-response study of N,N-dimethyltryptamine in humans. II. Subjective effects and preliminary results of a new rating scale. Arch Gen Psychiatry. 1994;51 (2): 98-108.

25. Kjellgren A, Eriksson A, Norlander T. Experiences of Encounters with Ayahuasca -- "the Vine of the Soul”. J Psychoactive Drugs. 2009; 41 (4): 309-15.

26. Santos RG, Landeira-Fernandez J, Strassman RJ, Motta V, Cruz APM. Effects of ayahuasca on psychometric measures of anxiety, panic-like and hopelessness in Santo Daime members. J Ethnopharmacol. 2007; 112 (3): 507-13.

27. Frecska E, Móré CE, VarghaA, Luna LE. Enhancement of creative expression and entoptic phenomena as after-effects of repeated ayahuasca ceremonies. J Psychoactive Drugs. 2012; 44(3): 191-9.

28. Bouso JC, González D, Fondevila S, Cutchet M, Fernández X, Ribeiro Barbosa PC, et al. Personality, Psychopathology, Life Attitudes and Neuropsychological Performance among Ritual Users of Ayahuasca: A Longitudinal Study. PLoS ONE. 2012; 7 (8): 1-13.

29. Gable RS. Risk assessment of ritual use of oral dimethyltryptamine (DMT) and harmala alkaloids. Addiction. 2007;102(1):24-34.

30. Guimarães dos Santos R. Safety and Side Effects 
of Ayahuasca in Humans-An Overview Focusing on Developmental Toxicology. J Psychoactive Drugs. 2013; 45 (1): 68-78.

31. Seguín CA. De la Medicina Folklórica. Medicinas Tradicionales y Medicina Folklórica. Lima: Fondo Editorial del Banco Central de Reserva del Perú; 1988. p. 57-98.

32. Valdivia Ó. El médico hechicero. Hampicamayoc Medicina Folklórica y su Substrato Aborigen en el Perú. Lima: Dirección Universitaria de Biblioteca y Publicaciones de la Universidad Nacional Mayor de San Marcos; 1975. p. 20-44.

33. Seguín CA. Valores humanos en psicoterapia. Acta Psiquiatr Psicol Am Lat.1967;13:121-6.

34. Seguín CA. What folklore therapy can teach us? Psychother Psychosom. 1973; 24: 293-302.

35. Chiappe M, Lemlij M, Millones L. La relación curandero-paciente. Sus implicancias médicosociales. Alucinógenos y Chamanismo en el Perú Contemporáneo. Lima: Ediciones El Virrey; 1985. p. 117-9.

36. Solanki MS, Zafar M, Rastogi R. Music as a therapy: Role in psychiatry. Asian J Psychiatr. 2013;6(3):1939.

37. Maratos AS, Gold C, Wang X, Crawford MJ. Music therapy for depression. Cochrane Database Syst Rev. 2008 (1):CD004517.

38. Trichter S, Klimo J, Krippner S. Changes in spirituality among ayahuasca ceremony novice participants. J Psychoactive Drugs. 2009; 41(2): 121-34.
39. Migdal L, Macdonald DA. Clarifying the relation between spirituality and well-being. J Nerv Ment Dis. 2013; 201(4): 274-80.

40. Michaux J. Hacia un sistema de salud intercultural en Bolivia: de la tolerancia a la necesidad sentida. En: Fernández G, editor. Salud e Interculturalidad en América Latina: Perspectivas Antropológicas. 1 ed. Quito: Ediciones Abya-Yala; 2004. p. 107-28.

41. Gastelumendi E. Ayahuasca-Another Deep Dive. 9th Conference of the INA Treating Neuropsychiatric Illnes: A Multidisciplinary Effort; Chicago, 2013.

42. Gonzales J. Du concept "hallucinogène" au concept "lucidogène" (aller-retour). In: Baud S, Ghasarian C, editors. Des Plantes Psychotropes Iniciations Thèrapies et Quêtes de Soi. Paris: Imago; 2010. p. 195-232.

43. Gastelumendi E. Ayahuasca: Current Interest in an Ancient Ritual. In: Miyoshi K, Morimura Y, Maeda $\mathrm{K}$, editors. Neuropsychiatric Disorders: Springer Japan; 2010. p. 279-86.

44. Samaja J. Subjetividad y Metodología. Epistemología de la Salud: Reproducción Social, Subjetividad y Transdisciplina. 1 ed. Buenos Aires: Editorial Lugar; 2009. p. 139-96.

Recibido: 23/10/2013 Aceptado: 21/03/2014 